\title{
Acute influence of mild hyperbaric oxygen at 1.25 atmospheres absolute with normal air on mitochondrial enzymes and PGC-1 $\alpha$ mRNA levels in rat skeletal muscle
}

\author{
Naoto Fujita ${ }^{1,2 *}$, Tomoka Tomioka ${ }^{2}$, Miharu Ono ${ }^{2}$ and Masataka Deie ${ }^{1,2}$ \\ ${ }^{1}$ Department of Musculoskeletal Functional Research and Regeneration, Graduate School of Biomedicine and Health Sciences, Japan \\ ${ }^{2}$ Division of Physical Therapy, Institute of Health Sciences, Faculty of Medicine, Hiroshima University, Japan
}

\begin{abstract}
Recent findings on hyperbaric oxygen treatment suggest potential usefulness in enhancing mitochondrial function and number in obese individuals with weightinduced osteoarthritis and lower-back pain. Therefore, if hyperbaric oxygen should enhance metabolic function of mitochondria in skeletal muscle, it might serve as an alternative to exercise to reduce obesity. The purpose of the present study was to investigate the effects of mild hyperbaric oxygen on mitochondrial content and biogenesis in skeletal muscle. Eight-week-old male Wistar rats were randomly assigned to hyperbaric-oxygen or non-hyperbaric-oxygen (control) groups. Rats in the hyperbaric-oxygen and control groups were exposed to 1.25 atmospheres absolute and normal atmospheric pressure, both with normal air, respectively. At 1 hof exposure to mild hyperbaric oxygen, the extensor digitorum longus and soleus muscles were removed and analyzed. In both the muscles, regardless of the muscle cell type, the activities of citrate synthase and succinate dehydrogenase were not altered due to exposure to the mild hyperbaric oxygen. Moreover, the expression level of peroxisome proliferator-activated receptor gamma coactivator 1 alpha (PGC-1 $\alpha$ ) protein remained unaltered; however, the mRNA level for PGC-1 $\alpha$ was significantly higher in the hyperbaric-oxygen group than in the control group. These results suggest that although mild hyperbaric oxygen does not enhance mitochondrial content acutely, regular treatment might lead to mitochondrial biogenesis over time.
\end{abstract}

\section{Introduction}

Individuals with body mass index $\geq 30 \mathrm{~kg} / \mathrm{m}^{2}$ are classified as obese. Obesity is considered a major risk factor for chronic diseases such as diabetes, heart failure, and stroke [1-3]. Obese individuals store adipose tissue excessively as subcutaneous and abdominal fat, and enhancement of lipid metabolism is required to reduce obesity. During lipid metabolism, triacylglycerol in the adipocyte is hydrolyzed to glycerol and fatty acids by lipase, and these are released into the blood [4]. The fatty acid moiety is transported to the mitochondria and undergo $\beta$-oxidation and is utilized for mitochondrial respiration. Because lipid utilization predominantly occurs in the mitochondria, enhancement of mitochondrial respiration could be effective to decrease excess adipose effectively. Aerobic exercises such as running and cycling are recommended for the reduction of obesity because such exercises promote fatty acid release from adipocytes and enhance their availability in the mitochondria of skeletal muscle cells [5]. In addition to promoting glucose and fatty acid uptake, exercise induces mitochondrial biogenesis in the myocyte [6]. Lira et al. [7] discussed that regular exercise increases mitochondrial volume and activity of the myocyte and results in increased lipid metabolism. Regular exercise effectively reduces obesity by inducing acute lipid oxidation during exercise and enhancing mitochondrial biogenesis over time in the myocyte. Mitochondrial biogenesis is regulated by peroxisome proliferator-activated receptor gamma coactivator 1 alpha (PGC-1a), the signal is activated by regular exercise, in muscle function and insulin sensitivity has been reviewed in [7]. Overexpression of PGC-1 $\alpha$ can shift from incomplete to complete $\beta$-oxidation in muscle of dietinduced obesity [8]. Although PGC-1 $\alpha$ could mediate lipid metabolism of myocyte and favor a reduction in obesity, maintaining a regular exercise schedule is difficult for obese individuals because of weightinduced osteoarthritis and lower-back pain.

Hyperbaric oxygen causes hyperoxia in several tissues, and has been accepted recently as a complementary treatment to promote healing of bone fracture [9], articular cartilage injury [10], and skeletal muscle injury [11]. Hyperbaric-oxygen treatment can be easily applied to obese individuals to treat obesity. Previously, we had reported that mild hyperbaric oxygen at 1.25 atmospheres absolute (ATA) with normal air prevents adipocyte hypertrophy in obese rats [12]. Quintero et al. reported that hyperbaric treatment with 95\% oxygen increases the release of glycerol from adipocytes in vitro [13]. In addition to increased glycerol-release, they demonstrated that hyperoxia increases the production of reactive oxygen species (ROS). Elevation of oxygen partial pressure induced by hyperbaric treatment increases the production of ROS in various tissues [14]. ROS are important signaling molecules for mitochondrial biogenesis, and increased ROS production activates some pathways leading to mitochondrial biogenesis $[7,15,16]$ However, the effect of ROS production by hyperbaric treatment on mitochondrial biogenesis in skeletal muscle is still unclear. Should

Correspondence to: Naoto Fujita, Department of Musculoskeletal Functional Research and Regeneration, Graduate School of Biomedicine and Health Sciences, Hiroshima University, 1-2-3 Kasumi, Minami-ku, Hiroshima 734-8553, Japan, Tel: +81-82-257-5423, Fax: +81-82-257-5423; E-mail: fujitan@hiroshima-u.ac.jp

Key words: metabolic function, mild hyperbaric oxygen, mitochondria, skeletal muscle

Received: April 13, 2016; Accepted: April 22, 2016; Published: April 25, 2016 

rat skeletal muscle

hyperbaric oxygen promote lipid metabolism by muscle-mitochondrial biogenesis, it may serve as a useful alternative to exercise for obese individuals. The purpose of the present study was to investigate the early effects of mild hyperbaric oxygen on mitochondrial biogenesis in the skeletal muscle.

\section{Materials and methods}

\section{Experimental design}

This study was approved by the Institutional Animal Care and Use Committee of Hiroshima University (A13-132), and was carried out according to the Hiroshima University Regulations for Animal Experimentation. All experiments were conducted in accordance with the National Institute of Health (NIH) Guidelines for the Care and Use of Laboratory Animals (National Research Council, 1996).

Eight-week-old male Wistar rats $(\mathrm{n}=12)$ were used in the present study. All the rats were randomly assigned to hyperbaric-oxygen ( $\mathrm{HB}$, $\mathrm{n}=6$ ) or non-hyperbaric-oxygen control (Cont, $\mathrm{n}=6$ ) groups. Rats in the $\mathrm{HB}$ group were exposed to $1.25 \mathrm{ATA}(1266.59 \mathrm{hPa})$ with normal air for $1 \mathrm{~h}$. Cages housing the Cont-group rats were placed in a hyperbaric chamber at normal atmospheric pressure $(1013.27 \mathrm{hPa})$. All animals were housed in a controlled environment at a constant temperature of $22 \pm 2^{\circ} \mathrm{C}$. Food and water were provided ad libitum. The rats were sacrificed with an overdose of sodium pentobarbital at lhafter exposure to mild hyperbaric oxygen. The extensor digitorum longus and soleus muscles were removed immediately, frozen in liquid nitrogen-cooled isopentane, and stored at $-80^{\circ} \mathrm{C}$ until further analysis.

\section{Enzymatic activity analysis}

The muscle samples were homogenized in ice-cold homogenizing buffer containing $10 \mathrm{mM}$ HEPES ( $\mathrm{pH} 7.3$ ), $11.5 \%$ sucrose, $0.1 \%$ Triton $^{\mathrm{Tw}} \mathrm{X}-100,1 \mathrm{mM}$ dithiothreitol, and $5 \%$ protease inhibitor cocktail (25955-11, Nacalai Tesque, Kyoto, Japan). After centrifugation at $1,500 \times \mathrm{g}$ for $10 \mathrm{~min}$ at $4^{\circ} \mathrm{C}$, the supernatants were collected. The diluted homogenate was added to a reaction mix containing $1 \mathrm{mM}$ 5,5'-dithiobis-(2-nitrobenzoic acid) solution and $10 \mathrm{mM}$ acetylcoenzyme A solution in a cuvette. After incubation for $10 \mathrm{~min}$ at $25^{\circ} \mathrm{C}$, $10 \mathrm{mM}$ oxaloacetate solution was added to the cuvette, and the optical density of the reaction solution was measured by spectrophotometry.

\section{Histological analysis}

Serial transverse sections of thickness $10 \mu \mathrm{m}$ were obtained using a cryostat from the middle part of the muscle sample and were mounted on glass slides. The sections were stained for myofibrillar adenosine triphosphatase (ATPase) and succinate dehydrogenase (SDH). For ATPase staining, the sections were preincubated in barbital acetate buffer ( $\mathrm{pH} 4.2$ ) for $5 \mathrm{~min}$ at room temperature. Following washing with $0.1 \mathrm{M}$ barbital buffer containing $0.18 \mathrm{M} \mathrm{CaCl}_{2}$ ( $\mathrm{pH}$ 9.4) for $30 \mathrm{~s}$, the sections were incubated in $0.1 \mathrm{M}$ barbital buffer containing $0.18 \mathrm{M}$ $\mathrm{CaCl}_{2}$ and $4 \mathrm{mM} \mathrm{ATP} \mathrm{(pH} \mathrm{9.4)} \mathrm{for} 45 \mathrm{~min}$ at room temperature. The sections were then washed in $1 \% \mathrm{CaCl}_{2}$ and $2 \% \mathrm{CoCl}_{2}$ every $3 \mathrm{~min}$, and finally, in $0.01 \mathrm{M}$ sodium barbital. Following washing with distilled water, the sections were visualized using $1 \%$ ammonium sulfide. For SDH staining, the sections were incubated in $50 \mathrm{mM}$ phosphate buffer ( $\mathrm{pH}$ 7.5) containing $0.05 \%$ nitro blue tetrazolium and $50 \mathrm{mM}$ sodium succinate for $45 \mathrm{~min}$ at $37^{\circ} \mathrm{C}$. The sections were analyzed, and images were acquired on a microscope (BZ-9000, Keyence, Osaka, Japan). Using the images from the serial sections stained for ATPase and $\mathrm{SDH}$, the myocytes were categorized into types I, IIA, or IIB on the basis of a previous study [17]. Additionally, histochemical images of sections stained for SDH were digitized as grayscale images, and the SDH staining intensities were expressed as optical density values. The $\mathrm{SDH}$ staining intensity was quantified for 5 fields per muscle. At least 100 randomly selected values for SDH activity were analyzed for each muscle cell type. All measurements were carried out with the ImageJ software (NIH).

\section{Western blot analysis}

The muscle samples were homogenized in the buffer containing $50 \mathrm{mM}$ Tris- $\mathrm{HCl}(\mathrm{pH} 7.8), 0.15 \mathrm{M} \mathrm{NaCl}$, and $1 \%$ protease inhibitor cocktail (Nacalai tesque) at $4^{\circ} \mathrm{C}$ and centrifuged to removed debris, and the supernatants were collected. After measurement of total protein concentration, the homogenate were solubilized in the buffer containing $0.5 \mathrm{M}$ Tris- $\mathrm{HCl}$ (pH 6.8), $2 \%$ sodium dodecyl sulfate, $10 \%$ glycerol, $5 \%$ $\beta$-mercaptoethanol, and $0.005 \%$ bromophenol blue, and boiled for 10 min at $80^{\circ} \mathrm{C}$. Sixty micrograms of the protein sample was loaded on a $10 \%$ polyacrylamide gel electrophoresis at $40 \mathrm{~mA}$ and transferred to a polyvinylidene fluoride membrane at $200 \mathrm{~mA}$. After transfer, the membranes were blocked using a blocking reagent (Blocking one, Nacalai Tesque) for $60 \mathrm{~min}$ and incubated with anti-PGC-1a (1:200, sc-13067; Santa Cruz Biotechnology, TX, USA) antibody at $4^{\circ} \mathrm{C}$. After overnight incubation, the membranes were incubated for $60 \mathrm{~min}$ at room temperature with anti-rabbit (1:10,000, sc-2370; Santa Cruz Biotechnology) antibody conjugated to horseradish peroxidase. The signals were detected using a chemiluminescence detector (ECL Prime, GE Healthcare, NJ, USA) and were analyzed with an image reader (Versa Doc 5000, BioRad).

\section{Quantitative polymerase chain reaction (qPCR) analysis}

Total RNA was isolated from each muscle using TRIzol $^{\circ}$ reagent (15596-026, Invitrogen, Tokyo, Japan) and the synthesis of cDNA was carried out using the High-Capacity cDNA Reverse Transcription Kit (4374966, Applied Biosystems, CA, USA). qPCR were performed to quantify the expression level of PGC-1a mRNA (Rn00580241_m1, Applied Biosystems) and normalization gene 18S (Rn03928990_g1) with the TaqMan ${ }^{\circ}$ Gene Expression Assays in CFX96 ${ }^{\text {tw }}$ Real-Time PCR Detection System (BioRad).

\section{Statistical analysis}

Data were expressed as means \pm standard error. The significance of differences between the groups was evaluated using the independent $t$-test, and $P<0.05$ was considered statistically significant.

\section{Results}

After 1hof exposure to the mild hyperbaric oxygen, skeletal muscle was analyzed as described in Methods.

\section{CS activity of skeletal muscle}

There were no significant differences in the CS activities of both the extensor digitorum longus (Figure 1A) and soleus (Figure 1B) muscles, between the Cont and HB groups.

\section{SDH activity of myocyte}

ATPase- and SDH-staining revealed that the extensor digitorum longus muscles were composed of type I, IIA, and IIB cells (Figures 2A$2 \mathrm{D}$ ), and the soleus muscles were composed of type I and IIA cells (Figures $2 \mathrm{H}-2 \mathrm{~K}$ ). In both the extensor digitorum longus (Figures $2 \mathrm{E}-2 \mathrm{G}$ ) and soleus (Figures 2L,2M) muscles, regardless of the cell type, there was no significant difference in SDH activity between the Cont and HB groups. 
A

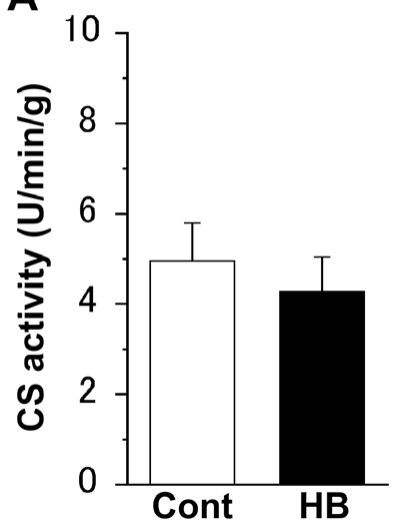

B

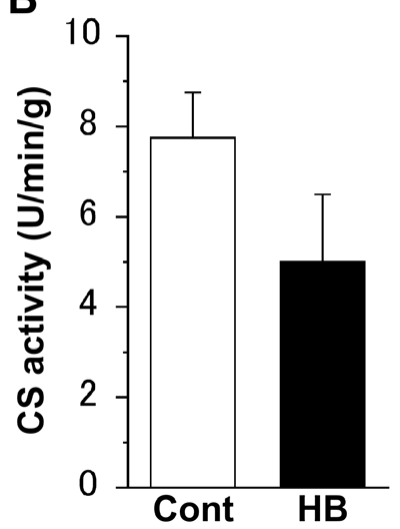

Figure 1. Citrate synthase (CS) activity of the extensor digitorum longus (A) and soleus (B) muscles. Values represent means \pm standard error (SE). $\mathrm{N}=6$ in all the groups. The following abbreviations are used for Figures 1-3. Cont: non-hyperbaric oxygen control; HB: hyperbaric oxygen.
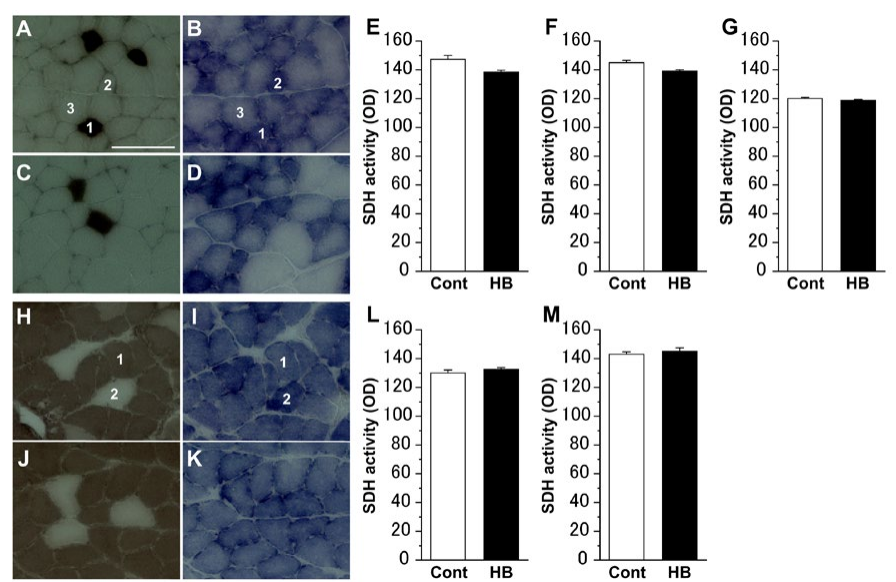

Figure 2. Succinate dehydrogenase (SDH) activity of myocyte in the extensor digitorum longus (A-D) and soleus ( $\mathrm{H}-\mathrm{K})$ muscles. Representative myocyte cross-sections stained for adenosine triphosphatase (ATPase) $(\mathrm{A}, \mathrm{C}, \mathrm{H}, \mathrm{J})$ and SDH (B, D, I, K) in the Cont (A, B, $\mathrm{H}, \mathrm{I})$ and $\mathrm{HB}(\mathrm{C}, \mathrm{D}, \mathrm{J}, \mathrm{K})$ groups. 1: type I cell; 2: type IIA cell; 3: type IIB cell. Scale bar $=100 \mu \mathrm{m}$. SDH activities of type I (E), IIA (F), and IIB (G) cells in the extensor digitorum longus muscle, and type I (L) and IIA (M) cells in the soleus muscle. Values represent means \pm SE. For each muscle cell type, over 100 myocytes were measured.

\section{Expression levels of PGC-1 $\alpha$ protein and mRNA}

There were no significant differences in the expression level of PGC-1 $\alpha$ protein in both the extensor digitorum longus (Figure $3 \mathrm{~A}$ ) and soleus (Figure $3 \mathrm{~B}$ ) muscles, between the Cont and HB groups. However, the expression level of PGC-1 $\alpha$ mRNA in the extensor digitorum longus muscle (Figure $3 \mathrm{C}$ ) in the $\mathrm{HB}$ group was approximately twice that in the Cont group, and the value in the soleus muscle (Figure 3D) was significantly higher in the HB group than in the Cont group.

\section{Discussion}

The activities of CS and SDH in the skeletal muscle tissue were not influenced by 1 htreatment with mild hyperbaric oxygen. CS is localized on the mitochondrial matrix, and it plays a key role in the tricarboxylic acid cycle [18]. CS catalyzes the formation of citrate from acetyl-coenzyme $\mathrm{A}$ and oxaloacetate. SDH is also known as succinateubiquinone oxidoreductase, and it is localized on the mitochondrial inner membrane [19]. SDH oxidizes succinic acid in the tricarboxylic acid cycle and reduces ubiquinone in the electron transport chain.

Using these enzymatic activities are indicators of mitochondrial metabolic function, the present study showed that $1 \mathrm{hmild}$ hyperbaric oxygen with mormal air has no effect on mitochondrial metabolism during exposure. Both oxygen uptake and consumption increase during exercise based on the intensity, resulting in enhanced metabolism and energy production [20]. Increased oxygen uptake during exercise is utilized to regenerate the ATP that was consumed by the exercise. Increased oxygen uptake also activates the adenosine monophosphateactivated protein kinase (AMPK) pathway $[7,15,16]$. However, the AMPK pathway might not have been activated by hyperbaric oxygen in the present study, because the animals remained still during exposure in the hyperbaric chamber. Similarly, Kurt et al. [21] have reported that hyperbaric oxygen increases ATP and decreases AMP concentrations in the skeletal muscle tissue, indicating that hyperoxia does not alter the AMPK pathway.

Although the expression level of PGC-1a protein was not altered by mild hyperbaric oxygen with normal air, the treatment enhanced the mRNA expression level of PGC-1a. PGC-1a is a transcriptional coactivator and a master regulator of the expression of genes involved in mitochondrial biogenesis [22]. Particularly in the skeletal muscle, PGC-1a regulates the energy metabolism with mitochondrial biogenesis by promoting the transcription of mitochondrial genes [23]. Matravadia et al. [24] showed that a single bout of exercise increases the expression level of PGC-1 only at the mRNA level. In their study, although one week of exercise did not alter the expression level of PGC$1 \alpha$ protein and other markers of mitochondrial content, four weeks of exercise significantly increased the expression levels of these markers at the protein level. Their results suggest that although single-bout or short-term exercise is insufficient to cause mitochondrial biogenesis, regular exercise induces mitochondrial biogenesis with increased PGCla protein. Additionally, Gutsaeva et al. [25] observed mitochondrial biogenesis at 10 days after regular treatment using hyperbaric oxygen in the hippocampus, but not at 5 days after the treatment, suggesting that mitochondrial biogenesis needs regular intervention. PGC-1a can suppress ROS in most cells due to induction of ROS-detoxifying enzymes [26]. If hyperbaric oxygen would produce ROS in myocyte, increased expression of PGC-1 $\alpha$ mRNA and mitochondrial biogenesis would result from the ROS production. Therefore, the finding that a single bout of mild hyperbaric oxygen with normal air enhanced the expression level of PGC-1 $\alpha$ mRNA in the present study lead us to surmise that regular treatment could induce mitochondrial biogenesis in the myocyte with upregulation of PGC-1 $\alpha$ protein.

In conclusion, although mild hyperbaric oxygen with normal air
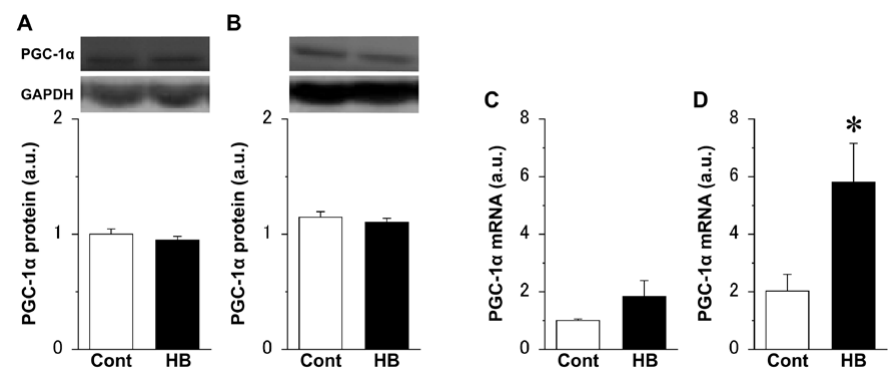

Figure 3. Expression levels of peroxisome proliferator-activated receptor gamma coactivator 1 alpha (PGC-1 $\alpha$ ) protein (A, B) and mRNA (C, D) in the extensor digitorum longus (A, C) and soleus (B, D) muscles and representative western blot images. Values were calculated as fold changes relative to the extensor digitorum longus muscle in the Cont group and represent means $\pm \mathrm{SE}$. * is significantly different from the Cont group, $P<$ $0.05 . \mathrm{N}=6$ in all the groups 
Fujita N (2016) Acute influence of mild hyperbaric oxygen at 1.25 atmospheres absolute with normal air on mitochondrial enzymes and PGC-1 $\alpha$ mRNA levels in rat skeletal muscle

did not enhance mitochondrial enzyme activities acutely in the skeletal muscle, the treatment led to increase of PGC-1a mRNA, suggesting its potential to induce mitochondrial biogenesis over time. Mitochondrial biogenesis are regulated by PGC- $1 \alpha$, along with activation of AMPKand ROS-dependent signaling pathways $[7,14,15]$. However, it is unlikely that exposure to hyperbaric oxygen increases AMP by enhanced consumption of ATP. Therefore, increase of PGC-1 1 mRNA induced by the single bout of mild hyperbaric oxygen with normal air might be associated with mechanisms other than the AMPK pathway. This study suggested the efficacy of mild hyperbaric oxygen with normal air in promoting muscle mitochondrial biogenesis. Treatment with hyperbaric oxygen may help obese individuals lose weight. However, the study has some limitations. We have not analyzed downstream molecules of the ROS and AMPK pathways. Additionally, the experimental model was limited to investigating only the acute effects of the treatment. Further research is required to determine the effect of mild hyperbaric oxygen with normal air on mitochondrial biogenesis in the myocyte over long periods.

\section{References}

1. Bastien M, Poirier P, Lemieux I, Després JP (2014) Overview of epidemiology and contribution of obesity to cardiovascular disease. Prog Cardiovasc Dis 56: 369-381. [Crossref]

2. Brixner DI, Bron M, Bellows BK, Ye X, Yu J, et al. (2013) Evaluation of cardiovascular risk factors, events, and costs across four BMI categories. Obesity (Silver Spring)21: 1284-1292. [Crossref]

3. Pi-Sunyer FX (1999) Comorbidities of overweight and obesity: current evidence and research issues. Med Sci Sports Exerc 31: S602-608. [Crossref]

4. Turner N, Cooney GJ, Kraegen EW, Bruce CR (2014) Fatty acid metabolism, energy expenditure and insulin resistance in muscle. J Endocrinol 220: T61-79. [Crossref]

5. Hegarty BD, Turner N, Cooney GJ, Kraegen EW (2009) Insulin resistance and fuel homeostasis: the role of AMP-activated protein kinase. ActaPhysiol (Oxf) 196: 129145. [Crossref]

6. Baldelli S, LettieriBarbato D, Tatulli G, Aquilano K, Ciriolo MR (2014) The role of nNOS and PGC-1 $1 \hat{\mathrm{I}} \pm$ in skeletal muscle cells. J Cell Sci127: 4813-4820. [Crossref]

7. Lira VA, Benton CR, Yan Z, Bonen A (2010) PGC-1alpha regulation by exercise training and its influences on muscle function and insulin sensitivity. Am J Physiol Endocrinol Metab 299: E145-161. [Crossref]

8. Koves TR, Li P, An J, Akimoto T, Slentz D, LLkayeva O, Dohm GL, Yan Z, Newgard CB, Muoio DM (2005) Peroxisome proliferator-activated receptor-gamma co-activator 1alpha-mediated metabolic remodeling of skeletal myocytes mimics exercise training and reverses lipid-induced mitochondrial inefficiency. J Biol Chem 280: 33588-33598. [Crossref]

9. Kawada S, Wada E, Matsuda R, Ishii N (2013) Hyperbaric hyperoxia accelerates fracture healing in mice. PLoS One8: e72603. [Crossref]

10. Ueng SW, Yuan LJ, Lin SS, Niu CC, Chan YS, et al. (2013) Hyperbaric oxygen treatment prevents nitric oxide-induced apoptosis in articular cartilage injury via enhancement of the expression of heat shock protein 70. J Orthop Res31: 376-384. [Crossref]

11. Fujita N, Ono M, Tomioka T, Deie M (2014) Effects of hyperbaric oxygen at 1.25 atmospheres absolute with normal air on macrophage number and infiltration during rat skeletal muscle regeneration. PLoS One 9: e115685. [Crossref]

12. Fujita N, Nagatomo F, Murakami S, Kondo H, Ishihara A, et al. (2012) Effects of hyperbaric oxygen on metabolic capacity of the skeletal muscle in type 2 diabetic rats with obesity. Scientific World Journal 2012: 637978. [Crossref]

13. Quintero P, González-Muniesa P, García-Díaz DF, Martínez JA (2012) Effects of hyperoxia exposure on metabolic markers and gene expression in 3T3-L1 adipocytes. J Physiol Biochem 68: 663-669. [Crossref]

14. Thom SR (2009) Oxidative stress is fundamental to hyperbaric oxygen therapy. J Appl Physiol (1985) 106: 988-995. [Crossref]

15. Radak Z, Zhao Z, Koltai E, Ohno H, Atalay M (2013) Oxygen consumption and usage during physical exercise: the balance between oxidative stress and ROS-dependent adaptive signaling. Antioxid Redox Signal 18: 1208-1246. [Crossref]

16. Sasaki T, Nakata R, Inoue H, Shimizu M, Inoue J, et al. (2014) Role of AMPK and PPAR $\hat{\mathrm{I}}^{3} 1$ in exercise-induced lipoprotein lipase in skeletal muscle. Am J Physiol Endocrinol Metab 306: E1085-1092. [Crossref]

17. Punkt K, Naupert A, Asmussen G (2004) Differentiation of rat skeletal muscle fibres during development and ageing. Acta Histochem 106: 145-154. [Crossref]

18. Wiegand G, Remington SJ (1986) Citrate synthase: structure, control, and mechanism. Annu Rev Biophys Biophys Chem15: 97-117. [Crossref]

19. Oyedotun KS, Lemire BD (2004)The quaternary structure of the Saccharomyces cerevisiae succinate dehydrogenase. Homology modeling, cofactor docking, and molecular dynamics simulation studies. J Biol Chem 279: 9424-9431. [Crossref]

20. Hirabara SM, Silveira LR, Abdulkader FR, Alberici LC, Procopio J, et al. (2006) Role of fatty acids in the transition from anaerobic to aerobic metabolism in skeletal muscle during exercise. Cell Biochem Funct24: 475-481. [Crossref]

21. Kurt B, Kurt Y, KarslioÄŸlu Y, Topal T, Erdamar H, et al. (2008) Effects of hyperbaric oxygen on energy production and xanthine oxidase levels in striated muscle tissue of healthy rats. J Clin Neurosci 15: 445-450. [Crossref]

22. Sano M, Tokudome S, Shimizu N, Yoshikawa N, Ogawa C, et al. (2007) Intramolecula control of protein stability, subnuclear compartmentalization, and coactivator function of peroxisome proliferator-activated receptor gamma coactivator 1alpha. J Biol Chem 282, 25970-25980. [Crossref]

23. Yan Z, Okutsu M, Akhtar YN, Lira VA (2011) Regulation of exercise-induced fiber type transformation, mitochondrial biogenesis, and angiogenesis in skeletal muscle. $J$ Appl Physiol (1985)110: 264-274. [Crossref]

24. Matravadia S, Martino VB, Sinclair D, Mutch DM, Holloway GP (2013) Exercise training increases the expression and nuclear localization of mRNA destabilizing proteins in skeletal muscle. Am J Physiol Regul Integr Comp Physiol 305: R822-831. [Crossref]

25. Gutsaeva DR, Suliman HB, Carraway MS, Demchenko IT, Piantadosi CA (2006) Oxygen-induced mitochondrial biogenesis in the rat hippocampus. Neuroscience 137: 493-504. [Crossref]

26. St-Pierre J, Drori S, Uldry M, Silvaggi JM, Rhee J, et al. (2006) Suppression of reactive oxygen species and neurodegeneration by the PGC-1 transcriptional coactivators. Cell 127: 397-408. [Crossref]

Copyright: (C2016 Fujita N. This is an open-access article distributed under the terms of the Creative Commons Attribution License, which permits unrestricted use, distribution, and reproduction in any medium, provided the original author and source are credited. 